\title{
Robot global path planning based on improved ant colony algorithm
}

\author{
Wang Rui ${ }^{1, a}$, Wang Jinguo ${ }^{2, b^{*}}$ (corresponding author), Wang $\mathrm{Na}^{3, \mathrm{c}}$ \\ ${ }^{1}$ Department of Information Engineering,Jilin Business and Technology College \\ China \\ ${ }^{2}$ Department of Urology, the First Hospital of Jilin University,China \\ ${ }^{3}$ Department of Anaesthesiology, the First Hospital of Jilin University, China

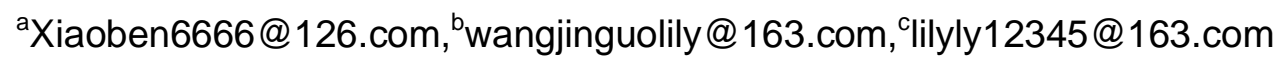

Key words: Mobile robot, Global Path planning, Ant colony algorithm

Abstract. In this paper, we present a path planning for mobile robot based on improved ant colony optimization algorithm. In order to increasing the algorithm's convergence speed and avoiding to fall into local optimum, we proposed updating the pheromone residues. Experiments show that this method is effective and feasible.

\section{Introduction}

The Italy scholar M.Dorigo proposed the ant colony algorithm, which is inspired by the foraging behavior of ants in nature in the early 1990s[1]. Using this new algorithm to solve the TSP problem, job-shop scheduling problem, the allocation problem, the experimental results are relatively satisfactory.Existing research results show that the ant colony algorithm is suitable for solving complex optimization problems, especially for discrete optimization problems[2].

Ant colony behavior has an information positive feedback phenomenon[3]. The more ants through the path, the higher the pheromone concentration is produced, the greater probability the next ants following. After a period of time, there will be the phenomenon of ants focused on the shortest path.

In the research of mobile robot technology, path planning technology is an important research area. It requires that The robot reaches its destination along a specific path. In the process, the mobile robot to avoid obstacles in the work environment, and to avoid collision with others. Therefore, path planning is an important part of the mobile robot navigation technology, and it is an important standard to judge the degree of intelligent robot.

\section{Ant colony algorithm model}

In the basic model of ant colony algorithm, the model establishment of ACS (ant colony system) and MMACS (Max-Min ant colony system) algorithm get a breakthrough research results. In the present research stage, the application and research of ant colony algorithm is based on these models. In the ACS algorithm, the process of the ants search the optimal pah using the local Pheromone. When all the ants searching activities end, using the global Pheromone, then we will strengthen the Pheromone on the best path the ants finding out. The ACS mainly carried out 3 improvements:

The transfer rules of ants are different. The formula that the ants select the next nodes is: 


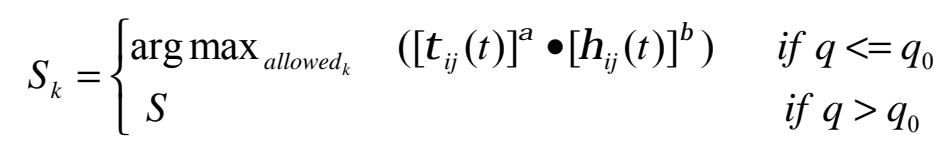

In which, $\mathrm{q}$ is random number between $[0,1]$. The $\mathrm{q} 0$ is a parameter between $[0,1]$. $\mathrm{S}$ is determined by formula (2)

$$
P^{k}(i, j)= \begin{cases}\frac{[\tau(i, j)]^{\alpha} \cdot[\eta(i, j)]^{\beta}}{\sum_{s \notin t a b u_{k}}[\tau(i, s)]^{\alpha} \cdot[\eta(i, s)]^{\beta}}, & \text { if } j \notin t a b u_{k} \\ 0 & \text { otherwise }\end{cases}
$$

In which, $\tau(i, j)$ is the pheromone concentration of the edge $(\mathrm{i}, \mathrm{j})$.

$\eta(i, j)=1 / d(i, j)$ is the heuristic information.d is the distance from city i to city $\mathrm{j}$.

$\alpha$ and $\beta$ reflect the relative importance of the pheromone and heuristic information.

$t a b u_{k}$ is the city list that the ant $\mathrm{K}$ has visited.

The method that determining the behavior of ants in the form of a certain random probability is a random probability selection rule. When the ant selects the next node, the algorithm will produce a random number $\mathrm{q}$ between $[0,1]$, and judge the relationship between $\mathrm{q}$ and $\mathrm{q}_{0}$, then determine the ants moving direction.

Global update rule is different. Global updates are only used for the best ant in every cycle, and no longer applies to all ants. The formula of its renewal rules is as follow:

$$
\begin{aligned}
& \tau(r, s)=(1-\alpha) \tau(r, s)+\alpha \Delta \tau(r, s) \\
& \Delta \tau(r, s)= \begin{cases}\frac{1}{L} & \text { if pathis optimal } \\
0 & \text { otherwise }\end{cases}
\end{aligned}
$$

In which, the $\mathrm{L}$ is the optimal route length has been calculated.

Add local update rule of adjustment amount of pheromone. After the ants completing the each transfer, update all the local pheromone on the path. The formula is as follow:

$$
\tau(r, s)=(1-\rho) \tau(r, s)+\rho \Delta \tau(r, s)
$$

In which, $\Delta \tau(r, s)$ can be 0 or a certain value.

Performance analysis. Although the ant colony algorithm has many advantages, but there are still some shortcomings. It is easy to solve the combination problem of the discrete domains, but is weak to solve the problem of continuous object optimization. Search time is too long, prone to stagnation phenomenon, and easy to fall into local optimal solution. When the searching to a certain stage, the algorithm converges to a local optimal solution, not further search the solution space, it will not find the global optimal solution.

\section{Algorithm improvement}

Setting $\rho_{0}$ of the ACS. In this paper, we use the state rule of the ACS as the formula (1). Because this method combines the exploration and the deterministic search together. In the initial stage of 
the algorithm, the $\rho_{0}$ is set to a large value, then go to a deterministic search and find the local optimal solution. In the middle of the iterative algorithm, the $\rho_{0}$ is set to a small value, which can expand the scope and probability of exploratory search. In the later period, the $\rho_{0}$ is returned to the initial value and the convergence rate is improved.

Setting the pheromone residues. In the front of this paper, we introduce the pheromone evaporation coefficient $\rho$, then $1-\rho$ is the Pheromone residual factor. We use $\rho_{r e}$ instead of pheromone residual factor for facilitate. In the practical operation, the greater the $\rho_{r e}$, the more Pheromone residual on the path, the smaller probability of path chosen that never be passed. It will reduce the global search ability of algorithm. If the $\rho_{r e}$ is smaller, the less pheromone residual is on the path, and it will block the convergence speed of the algorithm. So we set $\rho^{r e}$ value in a different section. In the initial stage, the $\rho_{r e}$ should be larger, so that the algorithm can find the optimal solution as soon as possible and it will increase the impact of the results. When discovery algorithm stagnation, $\rho_{r e}$ should be smaller, so that the algorithm can improve the global search, reduce the impact of pheromone, and prevent the emergence of the local optimum. Specific implementation formula is as follows:

$\rho_{r e}(t+1)=\left\{\begin{array}{lr}\max \left(\lambda \cdot \rho_{r e}(t), \rho_{\min }\right) & r=r_{\text {max }} \\ \rho_{r e}(t) & r \neq r_{\max }\end{array}\right.$

In which, $r$ is a continuous cycle number without evolution, $r_{\max }$ is a constant, $\lambda$ is between $(0,1)$ and is also a constant, $\lambda$ control the speed of $\rho_{r e}$ decay. $\rho_{\min }$ is a minimum value of $\rho_{r e}$. But Because the $\rho_{r e}$ once too small, it will affect the convergence rate. When the cycle number reaches the upper bound, the value of the pheromone residue factor is reduced. Then cycle number is cleared, restart the count. Such an iteration until $\rho_{r e}$ reaches the minimum value $\rho_{\min }$.

\section{Simulation experiment}

In order to verify the effectiveness of the algorithm, a large number of simulation experiments are carried out. Ant colony parameters are: $\alpha=1, \rho=0.9$. The ants number is $m=100$. The maximum iteration number is $\mathrm{nc}=50$. The results of the improved ant colony optimization for the mobile robot path planning is shown as figure1. 


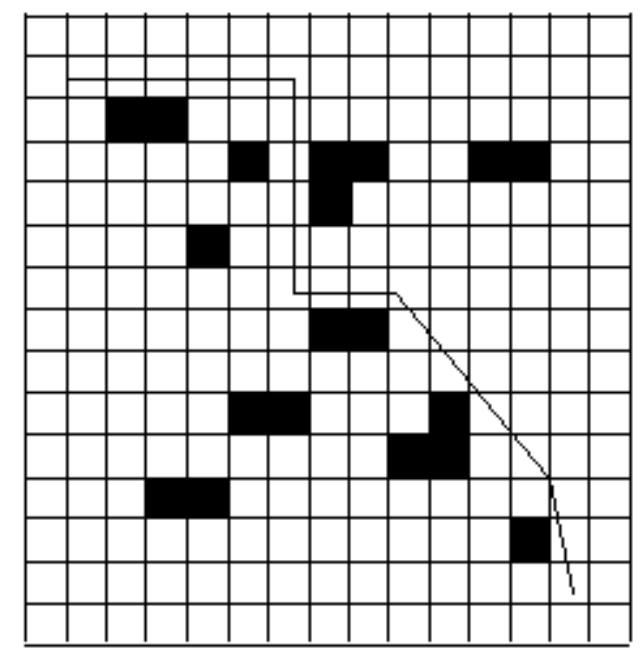

Fig. 1 the path planning of the improved ACO

\section{Conclusion}

Through many experiments, the algorithm can converge to the global optimum with great probability, and the convergence rate is very fast. But for the robot path planning in complex environment, the convergence speed of the algorithm is reduced, and it can also converge to the global optimal path. Experiments show that this method is effective.

\section{References}

[1] LI Lei, YE Tao, TAN Min. Present state and future development of mobile robot technology research[J]. Robot, 2002.

[2] Lim Kwee Kim,Ong Yew-Soon,Lim Meng Hiot,et al.Hybrid ant colony algorithms for path planning in sparse graphs[J].Soft Computing,2008.

[3]Thomas Stuztle. MAX-Min Ant System [J]. Furure Generation Computer System, 2000. 